\title{
Spherically Symmetric Solutions and Dark Matter on the Brane
}

\author{
Dan N. Vollick \\ Department of Physics \\ Okanagan University College \\ 3333 College Way \\ Kelowna, B.C. \\ V1V $1 \mathrm{~V} 7$
}

\begin{abstract}
It has recently been suggested that our universe is a three-brane embedded in a higher dimensional spacetime. In this paper I examine static, spherically symmetric solutions that satisfy the effective Einstein field equations on a brane embedded in a five dimensional spacetime. The field equations involve a term depending on the five dimensional Weyl tensor, so that the solutions will not be Schwarzschild in general. This Weyl term is traceless so that any solution of ${ }^{(4)} R=0$ is a possible four dimensional spacetime. Different solutions correspond to different five dimensional spacetimes and to different induced energy-momentum tensors on the brane. One interesting possibility is that the Weyl term could be responsible for the observed dark matter in the universe.
\end{abstract}




\section{Introduction}

It has recently been suggested that some of the extra dimensions required by string theory may be "large" [1, 2] or even infinite [3]. In the scenario proposed in [1], 2] the spacetime is $M^{(4)} \times K$, where $M^{(4)}$ is four dimensional Minkowski space and $K$ is a compact manifold. The size of the extra dimensions must be $\lesssim 5 \times 10^{-5} \mathrm{~mm}$ to be consistent with observations [4, 5]. In the Randall and Sundrum model [3] our three-brane is a domain wall separating two semi-infinite anti-de Sitter regions. In both scenarios the standard model fields are confined to the brane and gravity propagates in the bulk.

The Einstein field equations on the brane were derived by Shiromizu, Maeda, and Sasaki [6]. The effective four dimensional energy-momentum tensor contains terms involving stresses on the brane and a term involving the five dimensional Weyl tensor. The latter term carries information about the spacetime off the brane. Since the Weyl term is traceless any four dimensional spacetime with ${ }^{(4)} R=0$ gives rise to a three-brane world, without surface stresses, embedded in a five dimensional spacetime. In a recent paper Dadhich, Maartens, Papadopoulos, and Rezania [7] examined the Reissner-Nordstrom solution as an exact black hole solution without electric charge. The $g_{t t}$ component of the metric

$$
g_{t t}=1-\frac{2 m}{r}+\frac{Q}{r^{2}}
$$

contains the term $Q / r^{2}$, which does not arise from electric charge, but instead arises from projecting the gravitational field in the bulk onto the brane. In general relativity $Q=q^{2} \geq 0$, where $q$ is the black hole charge. Here $Q$ may be zero, positive or negative. It is important to note that there is an induced energy-momentum tensor on the brane if $Q \neq 0$.

In this paper I examine the static, spherically symmetric solutions that satisfy ${ }^{(4)} R=$ 0 . All of these solutions can be interpreted as spherically symmetric solutions on the brane with the Weyl term acting as the four dimensional energy-momentum tensor. Different solutions correspond to different five dimensional spacetimes and different Weyl terms. One interesting possibility that is discussed involves using the Weyl term to account for the observed dark matter in the universe.

\section{Spherically Symmetric Solutions}

The metric on the brane will be taken to be

$$
d s^{2}=-B(r) d t^{2}+A(r) d r^{2}+r^{2}\left(d \theta^{2}+\sin ^{2} \theta d \phi^{2}\right)
$$

and the equation ${ }^{(4)} R=0$ gives

$$
\frac{B^{\prime \prime}}{A B}-\frac{B^{\prime}}{2 A B}\left(\frac{A^{\prime}}{A}+\frac{B^{\prime}}{B}\right)+\frac{2}{r A}\left(\frac{B^{\prime}}{B}-\frac{A^{\prime}}{A}\right)+\frac{2}{A r^{2}}-\frac{2}{r^{2}}=0 .
$$


Of course, since we have one equation for two variables there is not a unique asymptotically flat solution. For a given $B(r)$ the resulting differential equation for $A(r)$ is

$$
f(r) \frac{A^{\prime}}{A}+\frac{2 A}{r^{2}}+g(r)=0
$$

where

$$
f(r)=\frac{B^{\prime}}{2 B}+\frac{2}{r}
$$

and

$$
g(r)=-\frac{B^{\prime \prime}}{B}+\frac{\left(B^{\prime}\right)^{2}}{2 B^{2}}-\frac{2 B^{\prime}}{r B}-\frac{2}{r^{2}}
$$

which can be integrated (in principle) to find $A(r)$. Here I will consider a few "types" of solutions to (3).

First consider solutions with $A(r)=B(r)^{-1}$. The resulting equation is

$$
r^{2} B^{\prime \prime}+4 r B^{\prime}+2(B-1)=0 .
$$

This is an Euler equation in $(B-1)$ and the general solution is

$$
A(r)^{-1}=B(r)=1+\frac{\alpha}{r}+\frac{\beta}{r^{2}}
$$

where $\alpha$ and $\beta$ are constants. Thus the Reissner-Nordstrom solution is the most general solution with $A(r)^{-1}=B(r)$.

Next consider spacetimes in which $B(r)$ takes the Schwarzschild form

$$
B(r)=1-\frac{2 m}{r}
$$

The general solution for $A(r)$ is

$$
A(r)=\left(1-\frac{2 m}{r}\right)^{-1}\left[\frac{3 m-2 r}{\lambda-2 r}\right]
$$

where $\lambda$ is a constant. Note that Schwarzschild spacetime is recovered if $\lambda=3 \mathrm{~m}$.

Unless $A(r)$ and $B(r)$ correspond to the Schwarzschild solution the brane will have a non-zero effective energy-momentum tensor given by [6]

$$
{ }^{(4)} T_{\mu \nu}=\frac{1}{8 \pi}{ }^{(5)} C_{\beta \rho \sigma}^{\alpha} \eta_{\alpha} \eta^{\rho} h_{\mu}^{\beta} h_{\nu}^{\sigma}
$$

where ${ }^{(5)} C_{\beta \rho \sigma}^{\alpha}$ is the five dimensional Weyl tensor evaluated on either side of the brane, $\eta_{\alpha}$ is normal to the brane, and $h_{\mu \nu}=g_{\mu \nu}-\eta_{\mu} \eta_{\nu}$ is the induced metric on the brane. The five dimensional spacetime off the brane has been examined perturbatively by Sasaki, 
Shiromizu, and Maeda [8], but is not known in general. It is therefore more useful to use

$$
{ }^{(4)} T_{\mu \nu}=-\frac{1}{8 \pi}{ }^{(4)} G_{\mu \nu} .
$$

It is also interesting to note that ${ }^{(4)} T_{\mu \nu}$ does not have to satisfy the weak energy condition [7, 9] and the spacetime may therefore contain negative energy densities.

Finally consider the weak field limit with

$$
R \simeq B^{\prime \prime}+\frac{2}{r}\left(B^{\prime}-A^{\prime}\right)+\frac{2}{r^{2}}\left(A^{-1}-1\right) .
$$

Now let $A=1+a$ and $B=1+b$ where $|a|,|b|<<1$. The field equation

$$
b^{\prime \prime}+\frac{2}{r}\left(b^{\prime}-a^{\prime}\right)-\frac{2}{r^{2}} a=0
$$

can be integrated to give

$$
a(r)=\frac{c_{1}}{r}+\frac{1}{2} r b^{\prime}(r),
$$

where $c_{1}$ is a constant.

One interesting possibility is that deviations from Schwarzschild geometry, produced by the Weyl term, could be responsible for the observed dark matter in the universe. Since galactic rotation curves become flat at large distances the Newtonian potential must contain a term proportional to $\ln r$. This implies that $b(r)$ has the form

$$
b(r)=-\frac{2 m}{r}+\alpha \ln r
$$

where $\alpha$ is a positive constant. To force $b(r) \rightarrow 0$ as $r \rightarrow 0$ we could replace $\ln r$ by $f(r) \ln r$ where $f(r) \simeq 1$ on galactic scales and $f(r) \ln r \rightarrow 0$ as $r \rightarrow \infty$. From (15)

$$
a(r)=\frac{c+m}{r}+\frac{1}{2} \alpha .
$$

The four dimensional energy-momentum tensor associated with the above solution is

$$
\begin{gathered}
T_{t t}=\frac{\alpha}{16 \pi r^{2}} \\
T_{r r}=\frac{\alpha}{16 \pi r^{2}}+\frac{m-c}{8 \pi r^{3}} \\
T_{\theta \theta}=\frac{c-m}{16 \pi r}
\end{gathered}
$$

and $T_{\phi \phi}=\sin ^{2} \theta T_{\theta \theta}$. Note that the energy density measured by static observers is positive if $\alpha>0$. 


\section{Conclusion}

In this paper I examined static spherically symmetric solutions of the equation ${ }^{(4)} R=0$. This equation arises in "brane-world" models that have zero surface stresses and a traceless source involving the Weyl tensor. Different solutions correspond to different five dimensional spacetimes and to different induced energy-momentum tensors on the

brane. One interesting possibility that was examined involved using the Weyl term to account for the observed dark matter in the universe.

\section{References}

[1] N. Arkani-Hamed, S. Dimopoulos, and G. Dvali, Phys. Lett. B429, 263 (1998), hep-ph/9803315

[2] I. Antoniadis, N. Arkani-Hamed, S. Dimopoulos, and G. Dvali, Phys. Lett. B436, 257 (1998), hep-ph/9804398

[3] L. Randall and R. Sundrum, hep-th/9906064

[4] S. Cullen and M. Perelstein, Phys. Rev. Lett. 83, 268 (1999), hep-ph/9903422

[5] L.J. Hall and D. Smith, Phys. Rev. D60, 085008 (1999), hep-ph/9904267

[6] T. Shiromizu, K. Maeda, and M. Sasaki, gr-qc/9910076

[7] N. Dadhich, R. Maartens, P. Papadopoulos, and V. Rezania, hep-th/0003061

[8] M. Sasaki, T. Shiromizu, and K. Maeda, hep-th/9912233

[9] D.N. Vollick, hep-th/0004064 